\title{
The role of Drosophila Merlin in spermatogenesis
} Natalia V Dorogova1, Elena M Akhmametyeva ${ }^{2}$, Sergei A Kopyl1, Natalia V Gubanova ${ }^{1}$, Olga S Yudina ${ }^{1}$, Leonid V Omelyanchuk ${ }^{1}$ and LongSheng Chang*2

\author{
Address: ${ }^{1}$ Institute of Cytology and Genetics, Russian Academy of Sciences, Novosibirsk, Russia and ${ }^{2}$ Center for Childhood Cancer, Research \\ Institute at Nationwide Children's Hospital and Department of Pediatrics, The Ohio State University College of Medicine, Columbus, Ohio, USA \\ Email: Natalia V Dorogova - natdorogova@rambler.ru; Elena M Akhmametyeva - akhmamee@ccri.net; Sergei A Kopyl - kopyl@bionet.nsc.ru; \\ Natalia V Gubanova - nat@bionet.nsc.ru; Olga S Yudina - olya_y@ngs.ru; Leonid V Omelyanchuk - ome@bionet.nsc.ru; Long- \\ Sheng Chang* - lchang@chi.osu.edu \\ * Corresponding author
}

Published: 10 January 2008

BMC Cell Biology 2008, 9:1 doi: $10.1|86 /| 47|-2| 2 \mid-9-1$
Received: 23 July 2007

Accepted: 10 January 2008

This article is available from: http://www.biomedcentral.com/|47/-2/2I/9/I

(c) 2008 Dorogova et al; licensee BioMed Central Ltd.

This is an Open Access article distributed under the terms of the Creative Commons Attribution License (http://creativecommons.org/licenses/by/2.0), which permits unrestricted use, distribution, and reproduction in any medium, provided the original work is properly cited.

\begin{abstract}
Background: Drosophila Merlin, the homolog of the human Neurofibromatosis 2 (NF2) gene, is important for the regulation of cell proliferation and receptor endocytosis. Male flies carrying a $M^{2} r^{3}$ allele, a missense mutation (Met ${ }^{177} \rightarrow$ le) in the Merlin gene, are viable but sterile; however, the cause of sterility is unknown.

Results: Testis examination reveals that hemizygous $\mathrm{Mer}^{3}$ mutant males have small seminal vesicles that contain only a few immotile sperm. By cytological and electron microscopy analyses of the $\mathrm{Mer}^{3}, \mathrm{Mer}^{4}$ (Gln ${ }^{170} \rightarrow$ stop), and control testes at various stages of spermatogenesis, we show that Merlin mutations affect meiotic cytokinesis of spermatocytes, cyst polarization and nuclear shaping during spermatid elongation, and spermatid individualization. We also demonstrate that the lethality and sterility phenotype of the $M \mathrm{rr}^{4}$ mutant is rescued by the introduction of a wild-type Merlin gene. Immunostaining demonstrates that the Merlin protein is redistributed to the area associated with the microtubules of the central spindle in telophase and its staining is less in the region of the contractile ring during meiotic cytokinesis. At the onion stage, Merlin is concentrated in the Nebenkern of spermatids, and this mitochondrial localization is maintained throughout sperm formation. Also, Merlin exhibits punctate staining in the acrosomal region of mature sperm.

Conclusion: Merlin mutations affect spermatogenesis at multiple stages. The Merlin protein is dynamically redistributed during meiosis of spermatocytes and is concentrated in the Nebenkern of spermatids. Our results demonstrated for the first time the mitochondrial localization of Merlin and suggest that Merlin may play a role in mitochondria formation and function during spermatogenesis.
\end{abstract}

\section{Background}

Spermatogenesis is a model that facilitates studies of the effect of gene mutations on mitosis, meiosis and the remodeling of many cell structures. During spermatogen- esis, primordial germ cells undergo an oriented mitotic division to replace themselves and to produce spermatogonia (reviewed in $[1,2]$ ). Each spermatogonium undergoes four rounds of mitotic division, generating 16 
spermatogonia that subsequently differentiate into spermatocytes within a cyst. Since the cytokinesis of mitotic divisions is incomplete, the spermatogonia are connected by ring channels. Then, all 16 spermatocytes go through two rounds of meiotic divisions, resulting in a cyst of 64 haploid, round-shaped spermatids. The meiotic cytokinesis is also incomplete so that the spermatids remain interconnected.

During the coalescence stage in early spermatids, the mitochondria aggregate to the side of the nucleus, where the centriole resides [2]. By the onion stage of spermatid differentiation, a dramatic transformation of the mitochondrial mass occurs. The individual mitochondria fuse into two giant mitochondria, which are arranged in a densely-packed sphere consisting of many layers of wrapped mitochondrial membranes [1]. This onion-like structure is termed the Nebenkern.

At the elongation stage, the flagellar axoneme elongates, resulting in a dramatic change in the shape of the spermatid [1]. The two interlocked subunits of the Nebenkern unfold and extend along with the growing axoneme. Despite the structural changes of the two mitochondrial derivatives, both mitochondrial subunits remain aligned and associated with axoneme. As spermatids begin to elongate, their heads, containing nuclei, remain aligned toward the testis wall and their tails are turned aside toward the testicular apex. Simultaneously, the cyst slides down along the testis wall, changing its shape from a disclike structure to a bundle of elongating spermatids with the nuclear regions of spermatids polarized toward the base of the testis [3]. Following the flagellar elongation, the spermatid nucleus transforms its shape from a spherical structure to a long, thin needle. Subsequently, the process of individualization is initiated by the formation of the individualization complex (IC), containing the actin cones at the head region of the spermatid bundle [4]. Individualization occurs in a cystic bulge, progressing along the entire length of the spermatid bundle. During individualization, membrane remodeling takes place, the channels connecting spermatids are destroyed, and syncytial organization of a cyst is lost [5]. Following coiling of the sperm bundle, mature sperm are released into the testis lumen and then pass into the seminal vesicle.

Although the spermatogenesis process has been well defined, only a limited number of genes whose mutations affect this process has been described, and the role of their protein products are mostly unknown. $\mathrm{Mer}^{3}$, a mutation (Met ${ }^{177} \rightarrow$ Ile) in the gene encoding Drosophila Merlin, whose ortholog in human is named the Neurofibromatosis 2 (NF2) gene [6,7], leads to male sterility [8]. Male flies with a $\mathrm{Mer}^{3}$ allele are viable but sterile; however, the cause of sterility is unknown.
Merlin shares a high degree of homology to the ezrin, radixin, and moesin (ERM) proteins, which belong to the protein 4.1 superfamily, linking the actin cytoskeleton to the plasma membrane [9]. Interaction of the ERM proteins with the actin cytoskeleton is thought to be important for the determination of the cell-shape, cell adhesion, cell motility, cytokinesis, and intracellular signaling $[10,11]$. In addition to its interaction with the actin cytoskeleton, Merlin can bind to microtubules and regulate the microtubule cytoskeleton $[12,13]$. Studies in mammalian cells show that Merlin mediates contact inhibition of proliferation [14]. Merlin inactivation leads to tumor formation in several cell types in mammals [15].

Merlin is evolutionally conserved [16]. The Merlin homolog in Drosophila melanogaster shows extensive sequence homology to the human protein [17]. This similarity between the fly and human Merlin proteins extends over the entire amino acid sequences with the greatest similarity in the amino terminus of the FERM domain ( $F$ for protein 4.1) [9,16-18]. The homology between the fly and human Merlin proteins also exists in the carboxyl terminus, a region in which Merlin diverges from the ERMfamily members $[10,16]$.

The Drosophila Merlin gene (D-Mer) is located at the $18 \mathrm{D}$ $\mathrm{E}$ region of the $\mathrm{X}$ chromosome. D-Mer has been shown to regulate cell proliferation and survival through the Hippo signaling pathway [8,19-23]. In addition, Merlin promotes endocytosis of several membrane signaling receptors [24,25]. Furthermore, D-Mer is non-autonomously required to maintain polarity of posterior follicle cells in the oocyte and to limit their proliferation [26].

To better understand the cause of sterility in Merlin mutant flies, we carried out an extensive analysis of cellular events in spermatogenesis. We showed that the Merlin protein was concentrated in the mitochondrial derivatives, and that Merlin mutations affected meiosis, cyst polarization, nuclear shaping, and axoneme-Nebenkern association.

\section{Results \\ The sterility phenotype in Merlin mutants is rescued by the introduction of a wild-type Merlin gene}

The Mer ${ }^{3}$ allele carries a missense mutation (Met ${ }^{177} \rightarrow$ Ile), and male flies hemizygous for $\mathrm{Mer}^{3}$ are viable but sterile [8]. Upon examination of the testis, we noted that seminal vesicles from the $\mathrm{Mer}^{3}$ males were smaller than those from the control FM6 flies (compare Figure 1B with Figure 1A). In addition, very few sperm were found in the Mer ${ }^{3}$ seminal vesicles, and they were immotile, in contrast to those seen in the control FM6 siblings obtained from the same cross. Acetic acid/orcein staining showed that the Mer ${ }^{3}$ testis had fewer sperm heads in each bundle than the control 


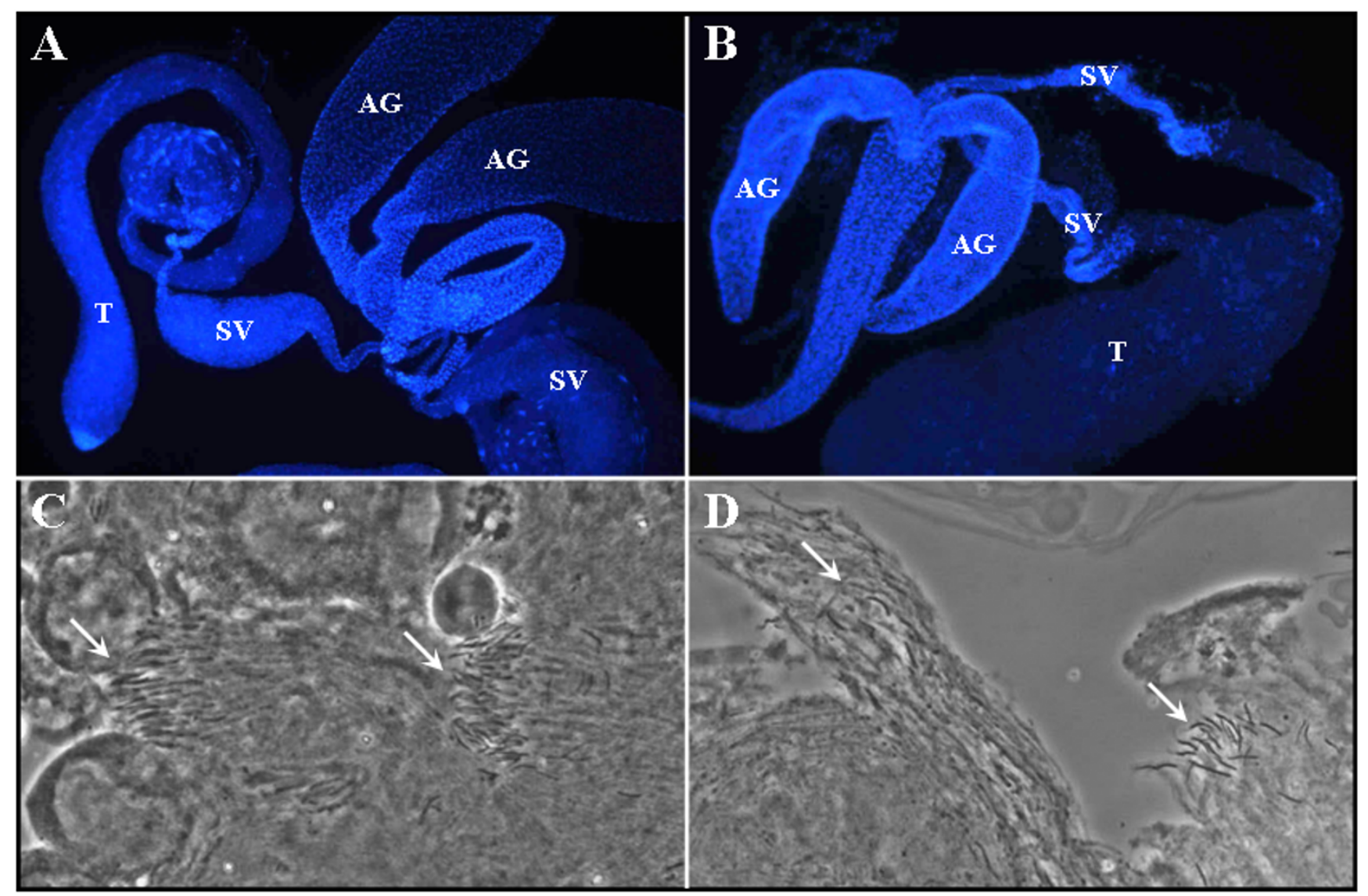

Figure I

Morphological examination of the testis tissues from young wild-type (panels A and C) and Mer ${ }^{3}$ (panels B and D) males. The control FM6 and Mer ${ }^{3}$ mutant males were obtained according to Materials and Methods. The DAPI-stained testis from the control male contained a bulging seminal vesicle (SV) (A). In contrast, a shriveled seminal vesicle was seen in the Mer ${ }^{3}$ testis (B). T, testis; AG, accessory gland. Acetic acid/orcein staining revealed that the testis tissue from the control male contained wellorganized sperm bundles (arrows) (C), while the Mer ${ }^{3}$ testis had fewer sperm in a more disorganized bundle (arrows) (D).

testis (compare Figure 1D with Figure 1C). Also, the sperm in the bundle were arranged in a more disorganized fashion.

Previously, LaJeunesse et al. [8] demonstrated that ectopic expression of a $\mathrm{Mer}^{+}$or $\mathrm{Mer}^{3}$ transgene using a ubiquitously-expressed Gal4 driver (T80-Gal4) in a $\mathrm{Mer}^{4}$ (Gln ${ }^{170} \rightarrow$ stop) background rescued $\mathrm{Mer}^{4}$ lethality. In addition, insertion of a cosmid construct $\left(P\left\{\cos \mathrm{Mer}^{+}\right\}\right)$, carrying the entire D-Mer gene, was capable of rescuing various Mer mutations. We conducted a similar experiment to test whether a $\mathrm{Mer}^{+}$transgene could rescue the sterility of the $\mathrm{Mer}^{4}$ allele. Table 1 shows that males carrying both $\mathrm{Mer}^{4}$ and $P\left\{\cos \mathrm{Mer}^{+}\right\}$were viable and fertile. To ensure germline expression of the $\mathrm{Mer}^{+}$or $\mathrm{Mer}^{3}$ transgene, we cloned the $\mathrm{Mer}^{+}$and $\mathrm{Mer}^{3}$ sequences into the pUASP vector, and used them to transform embryos. We showed that both pUASP-Mer ${ }^{+}$and pUASP-Mer ${ }^{3}$ could rescue the lethality of $\mathrm{Mer}^{4}$ mutation when ectopically activated by the Act5C-Gal4 driver (Table 1). However, only Mer+ overexpression restored the fertility of flies with the Mer ${ }^{4}$ mutation.

\section{The Merlin protein is dynamically redistributed during meiosis of spermatocytes and is concentrated in the Nebenkern of spermatids}

To understand the cause of sterility in the Merlin mutant flies, we studied the subcellular localization of the Merlin protein in the control FMG and $\mathrm{Mer}^{3}$ testes at various stages of spermatogenesis. In the cysts from the control testis, Merlin expression was detected in the cellular cortex of spermatocytes (Figure 2A), as seen in somatic tissues and earlier germ cells [17]. This cortical localization became more pronounced in spermatocytes during prometaphase and metaphase of meiosis (Figures $2 \mathrm{~B}$ and 2C). Merlin was found to redistribute, and more intense staining was observed in the area associated with the microtubules of the central spindle in telophase (Figure 
Table I: The ability of various transgenes to restore the viability and fertility of the $\mathrm{Mer}^{4}$ allele.

\begin{tabular}{|c|c|c|c|}
\hline Genetic cross & Males carrying $\mathrm{Mer}^{4}$ & Viability & Fertility \\
\hline \multirow{2}{*}{ 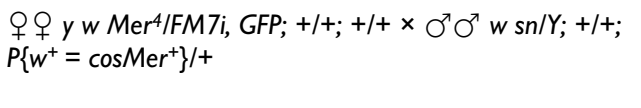 } & $y w M^{4} r^{4} / Y ;+/+; P\left\{w^{+}=\cos M e r^{+}\right\} /+$ & + & + \\
\hline & y w Mer $4 / Y ;+/+;+/+$ & - & - \\
\hline \multirow{4}{*}{$\begin{array}{l}\text { 우 y w } \mathrm{Mer}^{4} / \mathrm{M}-5, B w^{a} ;+/+;+/+\times O^{7} \sigma^{7} \text { y } w / Y ; \text { Act5C- } \\
\text { Gal4/If; } P\left\{w^{+}=\text {UASP-Mer } r^{+}\right\} / T M 6, T b\end{array}$} & y w Mer $4 / Y ;$ Act5C-Gal4/+; P\{w $w^{+}=$UASP-Mer +$\} /+$ & + & + \\
\hline & y w Mer/Y; Act5C-Gal4/+; +/TM6, Tb & - & - \\
\hline & y w Mer $4 / Y ;$ IfI+; TM6, Tb/+ & - & - \\
\hline & $y$ w Mer $4 / Y ; I f /+; P\left\{w^{+}=\right.$UASP-Mer $\left.{ }^{+}\right\} /+$ & - & - \\
\hline \multirow{4}{*}{$\begin{array}{l}\text { o } 9 \text { y w } \mathrm{Mer}^{4} / M-5, B w^{a} ;+/+;+/+\times O^{7} \sigma^{7} \text { y } w / Y ; \text { Act5C- } \\
\text { Gal4/If; } P\left\{w^{+}=\text {UASP-Mer } 3\right\} / T M 6, T b\end{array}$} & y w Mer $4 / Y ;$ Act5C-Gal4/+; P\{w $w^{+}=$UASP-Mer 3$\} /+$ & + & - \\
\hline & y w Mer4/Y; Act5C-Gal4/+; +/TM6, Tb & - & - \\
\hline & y w Mer $4 / Y ;$ Ifl+; TM6, Tb/+ & - & - \\
\hline & $y$ w Mer $4 / Y ; \mid f /+; P\left\{w^{+}=\right.$UASP-Mer 3$\} /+$ & - & - \\
\hline
\end{tabular}

2D). During cytokinesis, intense Merlin staining remained associated with the microtubules but the intensity was less in the region of the contractile ring (Figure 2E). A similar Merlin distribution pattern was seen during the second meiotic division (data not shown). At the onion stage, Merlin was concentrated in the Nebenkern, a specialized structure formed by the fusion of mitochondria during spermatid differentiation (Figure $2 \mathrm{~F}$ ). This intense Merlin staining in the Nebenkern remained throughout the comet stage of spermatid elongation, during which the Nebenkern split into two parts (Figure 2G). Note that concentrated Merlin immunoreactivity was clearly seen in the two subunits of the Nebenkern in the spermatid (insert in Figure 2G). In the control cyst containing mature sperm, Merlin staining continued to be present in the elongated Nebenkern (Figure 2H). In addition, Merlin was seen as a bright punctate dot in the acrosomal region, a Golgi apparatus-derived structure developed over the anterior part of the sperm's head. We also performed a similar immunostaining on the $\mathrm{Mer}^{3}$ testis. Although we could detect Merlin staining in the $\mathrm{Mer}^{3}$ cyst at the comet stage, the $\mathrm{Mer}^{3}$ spermatid nuclei were scattered throughout the cyst, and the arrangement of spermatids appeared irregular (Figure 2I). The ability of the antibody to detect Merlin staining in the $\mathrm{Mer}^{3}$ cyst suggests that the missense mutation in $\mathrm{Mer}^{3}$ did not affect antibody recognition. Using the same antibody, LaJeunesse [8] previously detected a similar cortical localization of Merlin in both the wild-type and $\mathrm{Mer}^{3}$ imaginal discs. However, the $\mathrm{Mer}^{3}$ mutation clearly affects Merlin function as the spermatids in the $\mathrm{Mer}^{3}$ cyst were poorly arranged.

\section{Merlin mutations affect meiotic cytokinesis of spermatocytes, cyst polarization and nuclear shaping during spermatid elongation, and spermatid individualization}

Next, we examined if there were any abnormalities during early steps of spermatogenesis in the $\mathrm{Mer}^{3}$ testis. We dis- sected testes from both the control and $\mathrm{Mer}^{3}$ mutant flies. Following staining with acetic acid/orcein, the testes were squashed and examined according to Ashburner [27]. Although we did not find any abnormalities during mitosis of spermatogonia or spermatocyte growth, we observed three types of abnormalities during meiosis of spermatocytes from the $\mathrm{Mer}^{3}$ testis, as compared with the control testis. The first type of abnormality is shown in Figure $3 \mathrm{~A}$, demonstrating a spermatid containing two nuclei of equal size and two Nebenkerns. This type of abnormality was likely caused by cytokinesis failure during the second meiotic division. The second type of abnormality is tripolar spindle in a spermatocyte going through the second meiotic division (Figure $3 \mathrm{~B}$ ). This result suggests incomplete cytokinesis in the previous meiotic division. The third type of abnormality is four-polar spindle in a spermatocyte undergoing the second meiotic division (Figures 3C-E). Note that a secondary $\mathrm{Mer}^{3}$ spermatocyte contained two pairs of telophase nuclei (Figure 3D). Each pair of nuclei was situated with its own spindle, and two spindles shared a common mid zone (Figure $3 \mathrm{C}$ and $3 \mathrm{E}$ ). This is in contrast with wild-type spermatocytes in telophase showing two daughter nuclei separate from each other along the central spindle (Figure 3F). The four-polar spindle represents another case of abnormal cytokinesis in the first meiotic division. It should be mentioned that we detected the first type of abnormality in about $5 \%$ of the mutant cysts, while the second and third types of abnormalities appeared less frequent.

Following meiosis, spermatid elongation ensues [1]. Prior to spermatid elongation, spermatid nuclei group at a defined area of the cyst wall in a process referred to as cyst polarization [3]. We noted that, at this stage, spermatid nuclei were detected as a group in the control cyst (Figure 4A). Intriguingly, spermatid nuclei in the $\mathrm{Mer}^{3}$ cyst were grouped in two locations (Figure 4B-E), and in some other cysts, nuclei appeared more scattered (Figure 4C-E). We also found a similar abnormality in nuclear grouping 


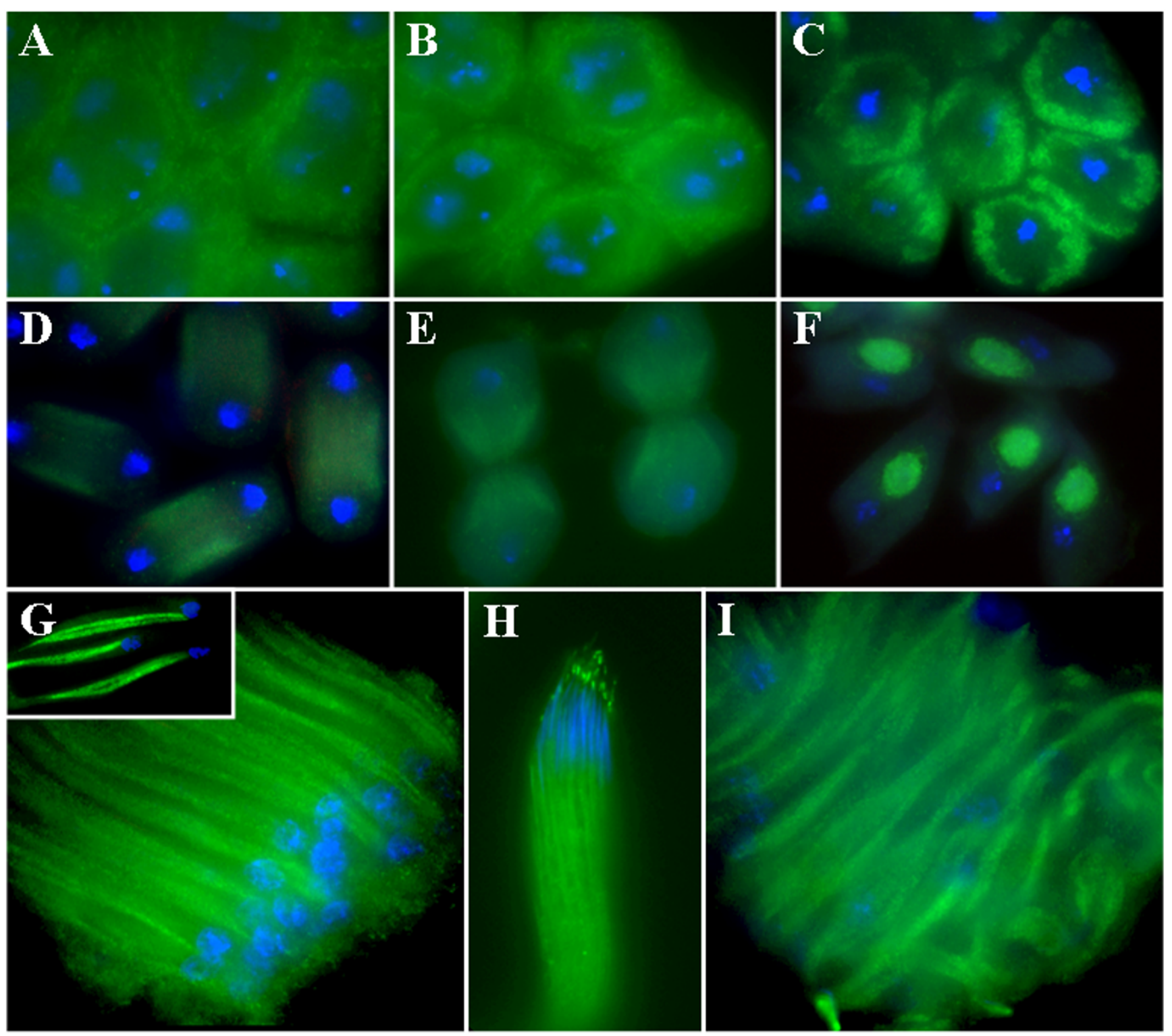

\section{Figure 2}

Intracellular distribution of the Merlin protein at various stages of spermatogenesis. In the control cyst, Merlin was detected in the cellular cortex of spermatocytes (A). In prometaphase (B) and metaphase (C) of meiosis, the cortical localization of Merlin became more evident. In telophase (D), Merlin redistributed and accumulated in the area associated with the microtubules of the central spindle. During cytokinesis $(E)$, Merlin staining remained associated with the microtubules but was less intense in the region of the contractile ring. In the onion-stage spermatids $(F)$, Merlin was highly concentrated in the Nebenkern. This localization pattern was maintained through the comet stage of spermatid elongation $(G)$. The insert in panel $G$ shows intense Merlin staining in the two subunits of Nebenkern in spermatids. In the control cyst, containing mature sperm, bright Merlin staining was also seen as a punctate dot in the acrosomal region $(\mathrm{H})$. Merlin staining was still detected in the Mer $^{3}$ cyst at the comet stage; however, sperm nuclei were scattered throughout the cyst, and the arrangement of spermatids was irregular (I).

in the $\mathrm{Mer}^{4}$ cyst. Although $\mathrm{Mer}^{4}$ is larva-lethal [19], we isolated some rare hemizygous $\mathrm{Mer}^{4}$ male pupae from the $y$ Mer ${ }^{4}$ Binsn stock, and a few of them were able to grow to pharates adults. When examining testis preparations from these $\mathrm{Mer}^{4}$ males, we detected spermatid nuclei arranged in two groups (Figures $4 \mathrm{~F}$ and $4 \mathrm{G}$ ) or in a scattered manner in all of the mutant cysts (Figure $4 \mathrm{H}$ ). Also, the cyst polarization defects were more frequently seen in $\mathrm{Mer}^{4}$ (about 90\%) than $\mathrm{Mer}^{3}$ (about 50\%) cysts. These results indicate that Merlin mutations affect cyst polarization. 

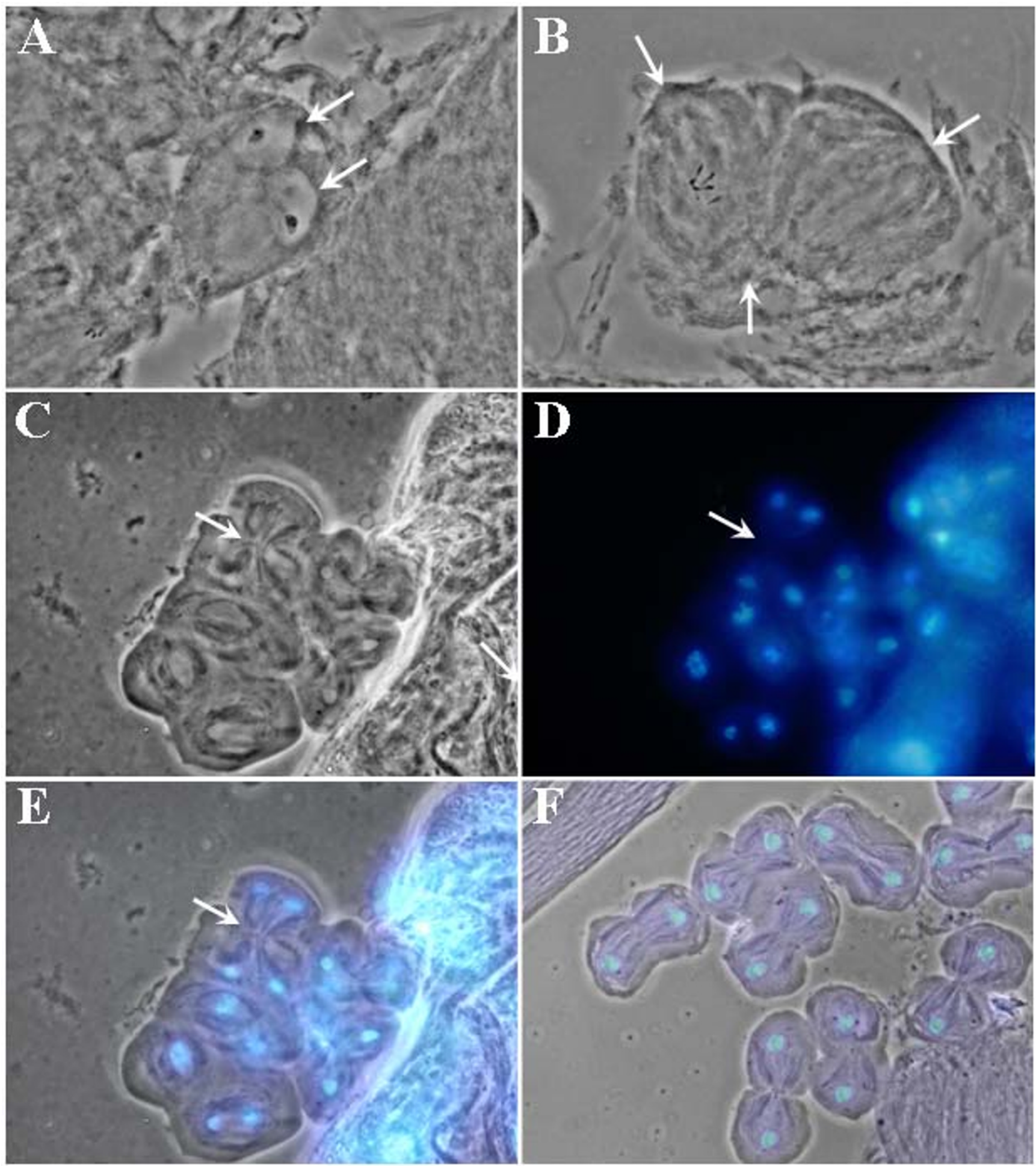

\section{Figure 3}

Abnormalities observed during meiosis of $\mathrm{Mer}^{3}$ spermatocytes: a spermatid containing two nuclei of equal size (arrows) and two Nebenkerns (A), tripolar spindle in a spermatocyte going through the second meiotic division (B), and four-polar spindle in a spermatocyte undergoing the second meiotic division (C-E). Panel C shows the phase contrast image, panel D the DAPIstained nuclei, and panel E the merged image. Arrow points to the central spindle midzone [29]. For comparison, wild-type meiotic cells in telophase were shown (F). 


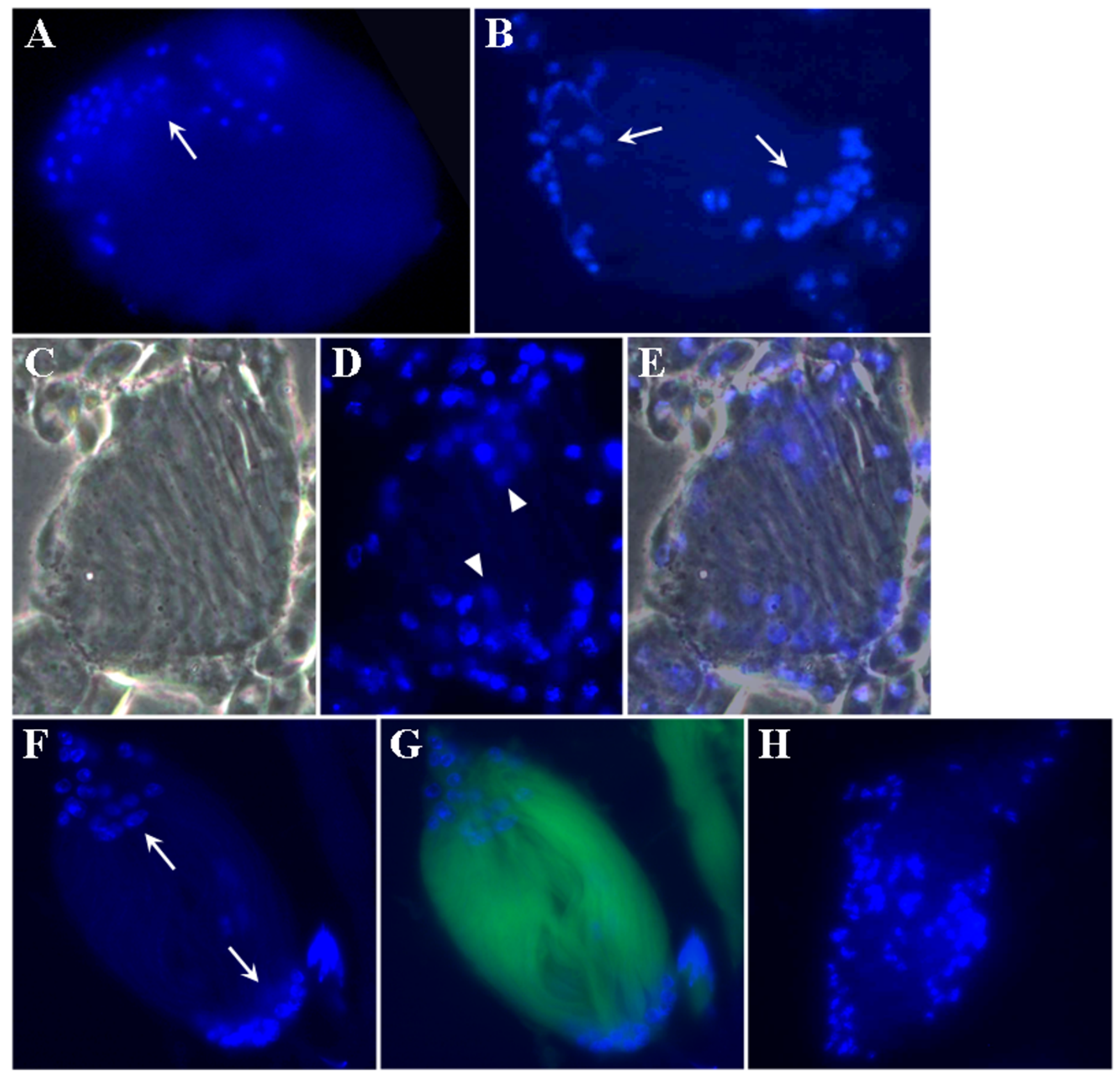

\section{Figure 4}

Difference in nuclear grouping during cyst polarization between the control and Merlin mutant spermatids. Dissected testes from the control FM6 (A), Mer ${ }^{3}(\mathrm{~B}-\mathrm{E})$, and $\mathrm{Mer}^{4}(\mathrm{~F}-\mathrm{H})$ males were stained with DAPI and examined as described before. Note that while the spermatid nuclei were grouped in one area (arrow) of the control cyst (A), the spermatid nuclei in the $M r^{3}$ (B$\mathrm{E})$ and $\mathrm{Mer}^{4}$ (F-G) cysts were seen as two diffuse groups (arrows). In some cases, the spermatid nuclei were scattered in the mutant cyst $(\mathrm{H})$. Panel $\mathrm{C}$ shows a phase-contrast image of a $\mathrm{Mer}^{3}$ cyst, panel D displays the same cyst stained with DAPI, and panel $E$ represents a merged image. The cyst shown in panels $\mathrm{F}$ and $\mathrm{G}$ was obtained from a male carrying the $M e r^{4}$ mutation and a GFP marker as described in Materials and Methods.

The final stage of spermatogenesis is the process of individualization, followed by sperm coiling $[1,2]$. The individualization process is initiated at the head of the spermatid cyst, and involves the formation and move- ment of the actin cones from the head region of the spermatid bundle to the caudal end [4]. Analogous to the previous finding, we detected the actin cones, which moved caudally as a bundle in the control cyst (Figures 
$5 \mathrm{~A}-\mathrm{D})$. Note that the sperm heads were grouped in one end of the control cyst, consistent to that seen at the cyst polarization stage (Figures $5 \mathrm{~B}$ and $5 \mathrm{D}$ ). Also, the sperm heads became needle-shaped. However, we observed that the sperm nuclei in the Mer ${ }^{3}$ cyst appeared scattered (Figure 5E-H) and had variable morphology; some were round, while others were needle shape (Figure 5I). Unlike the control cyst, the $\mathrm{Mer}^{3}$ cyst had the actin cones located
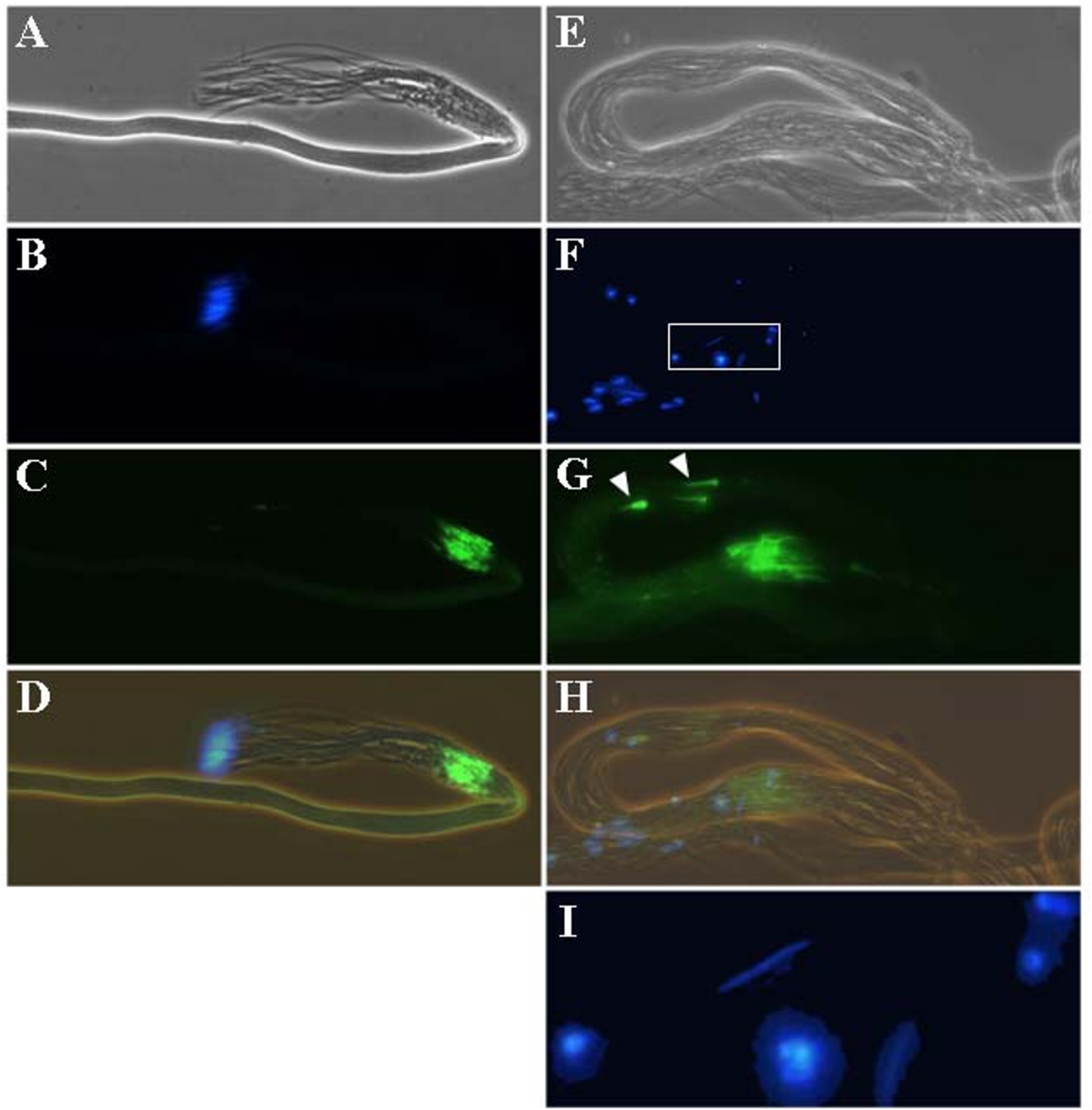

\section{Figure 5}

Spermatid individualization in the control (A-D) and $\mathrm{Mer}^{3}$ (E-I) cysts. Panels A and E illustrate the phase-contrast view of a control or Mer ${ }^{3}$ cyst, respectively. Panels B and $\mathrm{F}$ show the location of the DAPI-stained sperm nuclei. Panels $\mathrm{C}$ and $\mathrm{G}$ display the sites (arrowheads) and orientation of the actin cones as visualized by FITC-conjugated phalloidin staining. Panels $D$ and $H$ represent merged images. Panel I is an enlarged view of the rectangular area denoted in panel $F$. 
at multiple sites (Figure 5G). Dispersed actin cones together with scattered nuclei were also found in the $\mathrm{Mer}^{4}$ cyst (data not shown).

\section{Merlin mutant cysts display abnormalities in Nebenkern- axoneme association}

Since we detected intense Merlin staining in the Nebenkern at the onion stage, we employed electron microscopy to further examine Nebenkern transformation from the structure containing two mitochondrial derivatives at the late stage of spermatid elongation into a configuration filled by electron dense material, called the paracrystalline body, at the end of the individualization stage [4]. Thin sections of testes from the control FMG, Mer ${ }^{3}$, and $\mathrm{Mer}^{4}$ males were analyzed under a transmission electron microscope. As shown in Figure 6A, we observed that at the late elongation stage, each spermatid in the control cyst contained one major and one minor mitochondrial derivative associated with one axoneme. A paracrystalline body could be seen within the major mitochondrial derivative. However, we found that some of the spermatids in the $\mathrm{Mer}^{3}$ cyst contained two paracrystalline bodies within the major mitochondrial derivative (Figure 6B). Also, some spermatids had two axonemes. Similarly, two paracrystalline bodies within the major mitochondrial derivative were frequently seen in the elongating spermatids of the $\mathrm{Mer}^{4}$ cyst (Figure 6C). It should be mentioned that, unlike the spermatids in the control cyst, which displayed cellcell contact, the spermatids in the $\mathrm{Mer}^{3}$ cyst were loosely arranged (Figures $6 \mathrm{~B}$ ), and those in the Mer ${ }^{4}$ cyst were grossly disorganized (Figure 6C). In addition, some cytoplasmic shedding was present in the $\mathrm{Mer}^{3}$ cysts (Figure $6 \mathrm{~B})$, and excessive amount of cytoplasmic fragments was seen in the Mer ${ }^{4}$ cyst (Figure 6C).

At the individualization stage, each of the 64 spermatids in the control cyst contained one axoneme associated with the major and minor mitochondrial derivatives (Figure 6D). Furthermore, the paracrystalline body almost filled the entire major mitochondrial derivative. While some spermatids in the $\mathrm{Mer}^{3}$ cyst at a similar developmental stage had a paracrystalline body-filled major mitochondrial derivative with axoneme, others containing two paracrystalline body-filled mitochondrial derivatives with abnormal shape or three paracrystalline body-filled mitochondrial derivatives together with one axoneme were also seen (Figure 6E). In addition, spermatids having one or two paracrystalline-filled mitochondrial derivatives but lacking axoneme were observed. Also, the spermatids remained loosely contacted with each other in the $\mathrm{Mer}^{3}$ cyst. The paired arrangement of axoneme with the mitochondrial derivatives was often lost in the spermatids of the $\mathrm{Mer}^{4}$ cyst at the individualization stage (Figure 6F). Spermatids with multiple paracrystalline body-filled mitochondrial derivatives but without axoneme, or with two axonemes, were found. Also, cytoplasmic fragmentation together with condensed cytoplasmic remnants and gigantic cytoplasmic bodies were also seen in the Mer ${ }^{4}$ cyst.

Consistent with previous observations $[1,2,4]$, we noted that mature spermatids in the control cyst had a substantial reduction in the amount of cytoplasm and a significant reduction in the size of the minor mitochondrial derivative at the end of the individualization stage. The major mitochondrial derivative is filled by a dark-staining paracrystalline material (Figure 6G). Each of the 64 individualized spermatids in the control cyst displayed a highly ordered axoneme-Nebenkern pair. In contrast, a gross disorganization in the arrangement of the individualized spermatids in the Mer ${ }^{3}$ cyst was detected (Figure $6 \mathrm{H}$ ). Although some spermatids exhibited the axonemeNebenkern pair, others appeared to be fused together or connected by a thin cytoplasmic extension. In addition, the boundary between the cysts was not evident, and each cystic area contained less than 64 spermatids. The most dramatic alteration was observed in the $\mathrm{Mer}^{4}$ cyst (Figure 6I). Although axoneme and Nebenkern could be found, very little cytoplasmic material was seen, and the cell-cell boundary could not be easily identified. Insert in Figure 6I illustrates that the structure of axoneme appeared to be intact. Despite this dramatic alteration, the $9+2$ microtubule-containing structure of axoneme appeared to be preserved in the Merlin mutant cysts, indicating that Merlin is not required for axoneme formation and elongation.

Taken together, our results show that Merlin mutations affect cytokinesis, cyst polarization, nuclear shaping, and spermatid individualization. The observation that Merlin is highly concentrated in the Nebenkern suggests that Merlin may pay a role in mitochondria formation and function during various stages of spermatogenesis.

\section{Discussion}

Spermatogenesis is a complicated developmental process, including mitosis, meiosis, cell shape changes, and remodeling of subcellular organelles from the nucleus to mitochondria $[1,2]$. All of these events appear to involve cytoskeleton reorganization. Merlin has been shown to interact with the actin cytoskeleton and participate in the regulation of cell proliferation, cell adhesion, cell motility, and intracellular signaling $[10,11,15]$. Merlin can also interact with microtubules and regulate microtubule cytoskeleton $[12,13]$. In addition, an interaction between Merlin and the myosin heavy chain has been reported [28]. Consistent with findings that Merlin interacts with key components of the cytoskeleton, our results showed that Merlin mutations affected meiotic cytokinesis, cyst polarization, nuclear shaping, and spermatid individualization during spermatogenesis. We also showed that the 

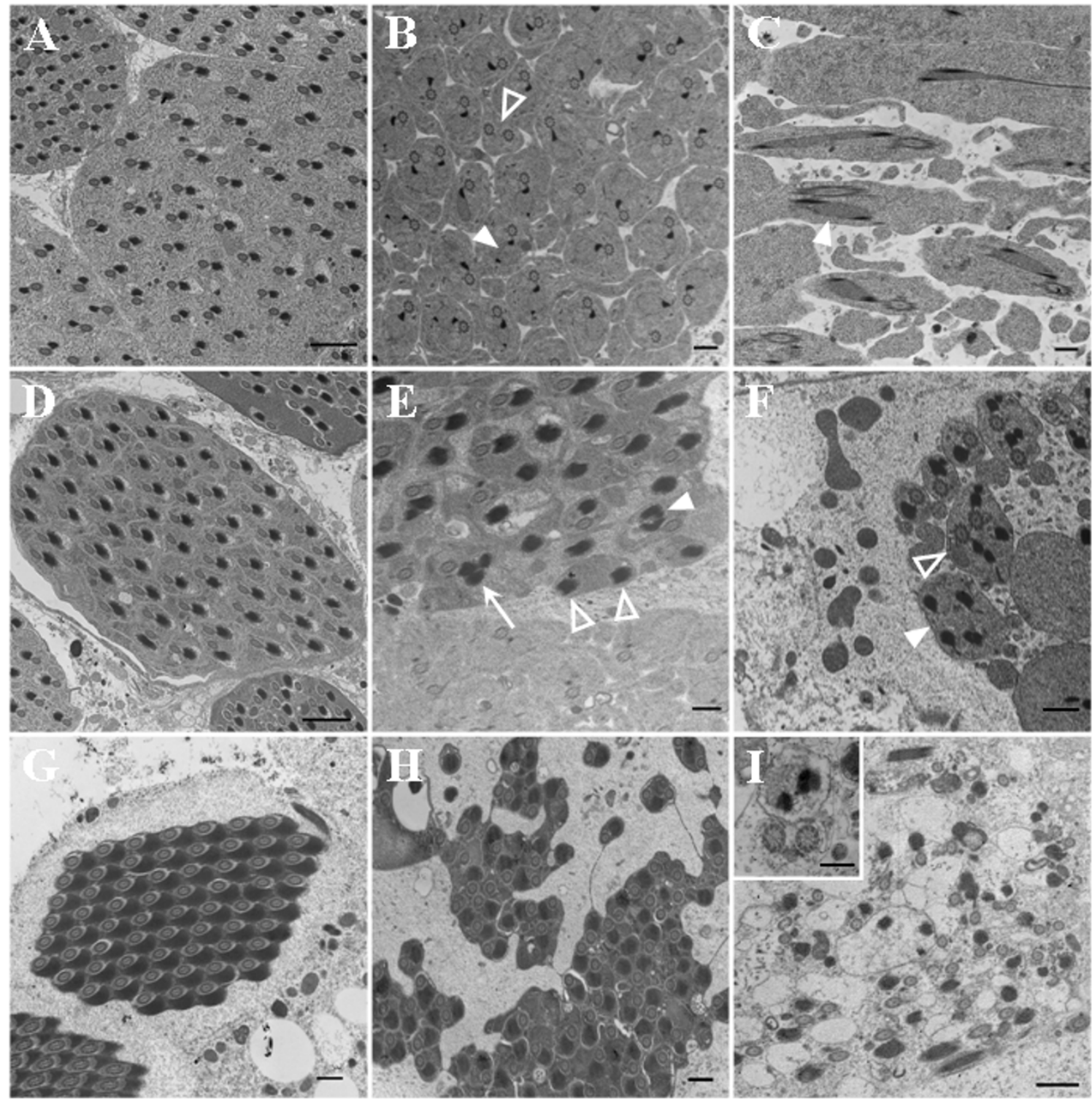

\section{Figure 6}

Ultrastructural analysis of the control and Merlin mutant cysts during the elongation and individualization stages. (A-C) Sections of the cysts from the control FM6 (A), Mer ${ }^{3}(B)$, or $\operatorname{Mer}^{4}(C)$ testis at the elongation stage. (A) A dark paracrystalline body was seen within the major mitochondrial derivative in the control spermatid. Bar $=2 \mu \mathrm{m}$. (B) Some of the spermatids in the $M^{3}{ }^{3}$ cyst contained two paracrystalline bodies (filled arrowhead points to one example). Also, some spermatids had two axonemes (open arrowhead). Bar $=0.5 \mu \mathrm{m}$. (C) Two paracrystalline bodies within the major mitochondrial derivative were frequently seen in the elongating spermatids of the Mer ${ }^{4}$ cyst. Bar $=0.5 \mu \mathrm{m}$. (D-F) Cysts from the control FM6 (D), Mer ${ }^{3}(\mathrm{E})$, or $\mathrm{Mer}^{4}$ (F) testis at the individualization stage. (D) The association of axoneme with the mitochondrial derivatives was seen in the spermatids of the control cyst. Bar $=2 \mu \mathrm{m}$. (E) Spermatids in the Mer ${ }^{3}$ cyst might contain two abnormally-shaped (filled arrowhead) or three (arrow) paracrystalline bodies together with one axoneme, or have two paracrystalline-filled Nebenkerns but without the axoneme (open arrowhead). Bar $=0.5 \mu \mathrm{m}$. (F) Spermatids with multiple Nebenkerns with (open arrowhead) or without (filled arrowhead) axonemes together with cytoplasmic fragmentation were seen in the $\mathrm{Mer}^{4} \mathrm{cyst}$. Bar $=0.5 \mu \mathrm{m}$. (G-I) Cysts from the control FM6 (G), Mer ${ }^{3}(\mathrm{H})$, or $\mathrm{Mer}^{4}(\mathrm{I})$ testis at the late stage of individualization. (G) Individualized spermatids in the control cyst displayed a highly-ordered axoneme-Nebenkern pair. Bar $=0.5 \mu \mathrm{m}$. $(\mathrm{H})$ The spermatids in the $\mathrm{Mer}^{3} \mathrm{cyst}^{\mathrm{s}}$ were poorly arranged and some of them appeared to be fused together. Bar $=0.5 \mu \mathrm{m}$. (I) The Mer $\mathrm{cyst}^{4}$ showed a complete destruction of spermatid individualization, resulting in empty spermatids with or without axoneme or Nebenkern. Insert illustrates the structure of axoneme appeared to be intact. Bar $=1 \mu \mathrm{m}$. Bar in the insert $=0.2 \mu \mathrm{m}$. 
sterility phenotype of hemizygous male Merlin mutants could be rescued by the introduction of a wild-type Merlin gene.

The first abnormality that we found in the Merlin mutants during spermatogenesis is cytokinesis failure during meiosis of spermatocytes. Cytokinesis is the process of dividing the cytoplasm and separating two daughter cells. It involves the formation of a contractile ring and the central spindle, two interdependent structures that cooperatively interact throughout the process [29]. The Drosophila contractile ring is comprised of actin, non-muscle myosin II, the regulatory light chain of myosin II, and anillin. Intriguingly, we observed that the Merlin expression pattern was closely associated with the microtubules of the spindle during cytokinesis. Given the fact that Merlin interacts with microtubule, actin, and myosin heavy chain, it is possible that Merlin may be involved in the assembly of the spindle and membrane addition. Further colocalization analysis will be needed to better understand the role of Merlin in this meiotic process.

Studies in mammalian cells indicate that Merlin functions both as a growth and tumor suppressor [15]. In Drosophila, tumor suppressors often regulate cell proliferation in a tissue-specific manner [30]. Two genes previously reported to have tumor suppressor property in the male gonad are bam and $b c g n$. Mutations in these genes result in a large number of cells resembling those in early germline stages [30]. Mosaic analysis in the eye tissue has revealed that Merlin mutant clones over-proliferate relative to normal sister clones; however, no tumors were found in the homozygous Merlin mutant tissue [8]. This result indicates that Merlin mutations belong to the class of overgrowth mutations. Upon careful examination of the $\mathrm{Mer}^{3}$ and $\mathrm{Mer}^{4}$ testis tissues, we also did not find any tumors.

Cyst polarization involves nuclear migration to a defined area of the cyst wall [3]. Although specific details about cyst polarization are not understood, it is envisaged that nuclear migration requires the participation of the actin cytoskeleton. Since Merlin interacts and modulates the actin cytoskeleton and other cytoskeletal apparatus, Merlin mutations could affect nuclear migration and lead to spermatid nuclei in two disorganized groups or more scattered in the mutant cysts as we have observed. Alternatively, the presence of Merlin in the Nebenkern suggests that Merlin may play a role in cyst polarization through Nebenkern. Nevertheless, Merlin represents the first gene whose mutation affects cyst polarization in spermatogenesis. Previously, in a genetic screen for genes functioning in embryonic axis specification, MacDougall et al. [26] found that gurken (grk) mRNA localization is altered in Mer mutant embryos. Normally, Grk signal instructs about 200 follicle cells to adopt a posterior fate. In turn, the posterior follicle cells send a polarizing signal back to the oocyte. Consequently, it induces the reorganization of oocyte microtubules, determining the localization of different mRNA and oocyte nuclear migration in the oocyte. Despite a broad expression pattern of Merlin in the egg chamber [17], Merlin appears to be specifically required non-autonomously only in a small group of follicular cells to maintain the polarity of posterior follicle cells and to limit their proliferation [26]. This finding is consistent with our data showing Merlin also has a role in cyst polarization during the spermatid pre-elongation period.

It should be mentioned that Merlin mutations also affect nuclear shaping during spermatid elongation as we observed a few spermatids with a round but not needleshaped head in the mutant cysts. Recent studies [31-33] have suggested a possible contribution of acrosome to nuclear shaping because defective acrosome development leads to round-headed sperm in mice. The biogenesis of the acrosome, a derivative of the Golgi complex important for sperm-egg penetration, requires the formation of the transient microtubule-containing manchette caudally to the acrosome [34]. In addition, the assembly of an Factin-containing cytoskeletal plate, called acroplaxome, serves as an anchor for the developing acrosome to the nuclear envelope [35]. Given the well-established relationship between Merlin and the actin filament or microtubule, Merlin mutations may affect any of the cytoskeleton-mediated structures required for acrosome formation. It is interesting to note that we have detected intense Merlin staining in the acrosomal region, suggesting a possible role of Merlin in this organelle.

Actin is a major cytoskeletal component of the IC, and individualization is accomplished by the assembly of the cytoskeletal-membrane complex at the nuclear end of the cyst $[1,4]$. Our results showing scattered nuclei and dispersed actin cones found in the Merlin mutant cysts are consistent with the idea that spermatid nuclei provide a physical scaffolding for the assembly of the IC. Intriguingly, several Drosophila mutants with scattered nuclei, including $\mathrm{Chc}^{4}$ (Clathrin heavy chain), scat ${ }^{1}$ (scattered), $c b x^{05704}$ (crossbronx), EcR ${ }^{06410}$ (Ecdysone Receptor), also display the dispersed IC phenotype [4]. The gene Clathrin heavy chain has been shown to participate in a number of biological processes, including receptor-mediated endocytosis, neurotransmitter secretion, and sperm individualization. The gene scattered is involved in Golgi-to-vacuole transport, retrograde transport from endosome to Golgi, and spermatid individualization. The gene crossbronx, encoding a ubiquitin-protein ligase, is important for the ubiquitin cycle and spermatid individualization. The gene Ecdysone Receptor, whose protein product responds to hormone stimuli, is essential for embryonic development and organogenesis, including spermatid development. 
Curiously, Merlin has been shown to promote endocytosis of several signaling receptors $[24,25]$. However, how this set of genes is connected to the individualization process remains to be determined.

Spermatid individualization involves membrane remodeling and the outcome of this process is endowing each spermatid with its own plasma membrane and simultaneously removing most of the syncytial cytoplasm from between sperm tails as it proceeds $[33,36]$. At present, we do not know how Merlin mutations lead to excessive cytoplasmic remnants and poorly organized spermatids at the end of individualization. Although Merlin may have a role in membrane remodeling as previously suggested, it is possible that spermatogenesis is such an orchestrated process that perturbation in each stage results in specific abnormalities, which could subsequently affect the following events.

Merlin has been shown to localize underneath the plasma membrane at cell-cell junctions and other actin-rich sites $[10,11]$. The detection of a high concentration of Merlin protein in the Nebenkern at the onion stage and its maintenance throughout mature sperm formation imply a unique role of Merlin in mitochondria formation and function. The presence of two or multiple paracrystalline bodies in the major mitochondrial derivative of Merlin mutant spermatids could result from Merlin dysfunction, leading to such an abnormal Nebenkern structure. A similar abnormality has also been observed in the mutants defective in the gene fuzzy onion ( $f z o)$, encoding a GTPase [37] or rotund ( $r n$ ), coding for a Rac GTPase activating protein [38]). However, it is not known whether Merlin function links to these signaling molecules in regulating Nebenkern formation. One fundamental function that mitochondria provide is the production of ATP, which serves as an energy source. The Nebenkern structure is pivotal to sperm motility. Although rare sperm could still be found in the $\mathrm{Mer}^{3}$ testis, it is likely that without normal Merlin function, sperm motility is impaired.

It should be mentioned that Merlin is not the only growth suppressor whose loss results in male sterility. Like Merlin, the Tumor Suppressor for Lung Cancer 1 (TSLC1) protein, an immunoglobulin superfamily molecule predominantly expressed in the brain, lung and testis, plays important roles in cell adhesion and tumor invasion in mammals [39]. Interestingly, TSLC1-deficient mice also produce round spermatids and are sterile. Thus, it will be important to see whether Merlin deficiency in the testis has any effect on the fertility of mice.

\section{Conclusion}

Drosophila Merlin mutant flies are viable but sterile, and the sterility phenotype is rescued by the introduction of a wild-type Merlin gene. Merlin mutations affect meiotic cytokinesis of spermatocytes, cyst polarization and nuclear shaping during spermatid elongation, and spermatid individualization. The Merlin protein is enriched in the Nebenkern and this mitochondrial localization is maintained throughout sperm formation. These results suggest a role of Merlin in mitochondria formation and function during various stages of spermatogenesis. Further investigation of the action of Merlin in mitochondria is warranted.

\section{Methods \\ Fly stocks}

Flies were maintained at $25^{\circ} \mathrm{C}$ in standard cornmeal yeast-agar medium. Various Merlin mutant strains were generously provided by Rick G. Fehon at University of Chicago, Chicago, IL [40]. Hemizygous $\mathrm{Mer}^{3}$ males were taken from the strain $w \operatorname{Mer}^{3} P\{r y[+t 7.2]=n e o F R T\} 19 A /$ $F M 6, y B$. The FMG, $y B$ siblings and the $y w$ males from the stock $y w$ Pim 19A-FRT/TM6, Tb (abbreviated here as Pim) were used as controls. The strain $y w \operatorname{Mer}^{4} P\{r y[+t 7.2]=$ neoFRT $\} 19 A / F M 7 i, P\{w[+m C]=$ ActGFP $\} J M R 3$ was used as a source of $\mathrm{Mer}^{4}$ mutant individuals. The $w$ $s n^{3} l(1) 18 D E b^{3} P\{r y[+t 7.2]=n e o F R T\} 19 A ; P\{w[+m C]=$ $\left.\cos \mathrm{Mer}^{+}\right\} 3 /+$ strain with an insertion of a genomic fragment containing the entire Mer gene was also used. Transgenic strains carrying the pUAST-M $y c \mathrm{Mer}^{+}$or pUAST$\mathrm{MycMer}^{3}$ constructs have been described previously [8]. The strain, containing an insertion of a transposable element carrying the green fluorescent protein (GFP) tag inserted into the CG8351 gene, was kindly provided by Alain Debec of Université Pierre et Marie Curie, Observatoire Océanologique, Villefranche-sur-mer, France. This strain allowed labeling the cytoplasm of all cells uniformly during spermatogenesis. The strain $y w$; Ki Delta23 carrying endogenous transposase activity was a gift from the laboratory of Igor Zhimulev, Institute of Cytology and Genetics, Novosibirsk, Russia.

\section{Acetic acidlorcein and DAPI staining}

The females $w \operatorname{Mer}^{3} P\{r y[+t 7.2]=$ neoFRT $\} 19 A / F M 6, y B$ were mated with the males FMG, y $B / Y$ from the same stock. The resulting $\operatorname{Mer}^{3}$ male $y w \operatorname{Mer}^{3} P\{r y[+t 7.2]=$ neoFRT $\} 19 A / Y$ and control FMG, $y$ B/Y males were obtained and their testes were dissected. The squashed preparations of testes were performed according to Ashburner [27]. Briefly, testes were dissected in Hanks balanced salt solution (HBSS) and stained in a 1:1 mixture of $1 \%$ acetic acid/orcein and $1 \%$ acetic acid/carmine for 2 hours at room temperature. Stained testes were examined under phase-contrast optics of an Axiovert-200 microscope (Carl-Zeiss). For DAPI staining, dissected testis tissues were fixed in 3.7\% formaldehyde in Dulbecco's phosphate-buffered saline (PBS), $\mathrm{pH} 7.2$, and stained with 
DAPI $(1.5 \mu \mathrm{g} / \mathrm{ml})$ prior to visualization under the epifluorescence optics of an Axiovert-200 microscope.

\section{Transgenesis}

Genomic DNA were isolated from flies carrying the UAS$\mathrm{MycMer}^{+}$or UAS-MycMer ${ }^{3}$ insertion as described above and amplified by PCR to generate Merlin CDNAs as previously described [8]. The Merlin cDNAs were inserted into the pUASP vector at the SacII and XbaI sites. The DNA inserts in the plasmids were confirmed by restriction digestions and direct sequencing. Embryos with the genetic constitution $y$ w; Ki Delta2-3, carrying the endogenous transposase Delta2-3, were injected with the pUASP-M $y c \mathrm{Mer}^{+}$or pUASP-M $y c \mathrm{Mer}^{3}$ DNA. After reaching the adult stage, the injected flies were mated with $y w$ mating partners. The resulting $w^{+}$progeny were isolated and crossed to establish a strain carrying a transposition. A few independent insertions were obtained for each construct, and the presence of the Merlin coding sequence in the transposants was tested by PCR analysis of genomic DNA described above.

\section{Living cytology}

For the examination of sperm motility, seminal vesicles of male flies that had been alone for three days were isolated and checked for movement of sperm heads under Varel contrast optics of an Axiovert-200 microscope. For general spermatogenesis inspection, dissected testes were squashed in HBSS using coverslips as described by Fuller [2]. The unfixed preparations of live cysts were examined for the coiling process according to Cross and Shellenbarger [3].

\section{Antibody staining}

Dissected testes were placed onto poly-L-lysine coated slides. To isolate cysts, the dissected testes were pierced using a tungsten needle attached to a Narishigi micromanipulator. Slides with testis tissues attached were fixed in $3.7 \%$ formaldehyde in PBS, pH 7.2. After washing in PBS $10 \mathrm{~min}$ three times, fixed tissues were permeated with $1 \%$ Triton X-100 in PBS for 30 min and then pretreated with the blocking solution containing $1 \%$ non-fat dry milk in PBS. Pretreated tissues were incubated with a guinea pig anti-Merlin antibody (1:6000 dilution; [17]) or a mouse anti- $\alpha$-tubulin antibody (1:500 dilution; Sigma Chemicals) overnight. After washing with PBS three times, a secondary antibody conjugate (Alexa 488-conjugated antiguinea pig IgG [1:700 dilution], Alexa 568-conjugated anti-mouse IgG [1:200 dilution, or a FITC-conjugated anti-mouse IgG [1:50 dilution]) was added for 2 hours at room temperature. To visualize actin filaments, FITC-conjugated phalloidin (1:50 dilution; Molecular Probe) was used. In some experiments, nuclei were stained with DAPI $(1.5 \mu \mathrm{g} / \mathrm{ml})$. After staining, the slides were mounted in Mowiol with $10 \%$ DABCO and examined under the epif- luorescence or phase-contrast optics of an Axiovert-200 or an Olympus BX50 microscope.

\section{Electron microscopy}

Dissected testes were fixed in 2\% glutaraldehyde in PBS, $\mathrm{pH} 7.4$, for 2 hours and then treated with $1 \%$ osmium tetraoxide in PBS for 1 hour. Treated tissues were stained with $1 \%$ uranyl acetate at $4{ }^{\circ} \mathrm{C}$ overnight. Following dehydration in ascending ethanol solutions, stained tissues were embedded in Agar-100, mounted in the block, and polymerized at $60^{\circ} \mathrm{C}$ for two to three days. Ultrathin sections were prepared and contrasted by incubating in $1 \%$ uranyl acetate and lead citrate, and examined using a Hitachi H7650 or a JEOL 1000SX transmission electron microscope.

\section{Abbreviations \\ IC, individualization complex}

ERM, the ezrin, radixin, and moesin proteins

NF2, the Neurofibromatosis 2 gene

FERM, protein 4.1, ezrin, radixin, and moesin

D-Mer, the Drosophila Merlin gene

grk, the gurken gene

Grk, the Gurken protein

Chc, the Clathrin heavy chain gene

scat, the scattered gene

$c b x$, the crossbronx gene

$E c R$, the Ecdysone Receptor gene

fzo, the fuzzy onion gene

$m$, the rotund gene

TSLC1, the Tumor Suppressor for Lung Cancer 1 protein

GFP, green fluorescent protein

HBSS, Hanks balanced salt solution

PBS, phosphate-buffered saline

\section{Authors' contributions}

NVD performed immunostaining analysis, EMA and NVG carried out electron microscopy, SAK conducted genetic crosses, OSY generated pUASP constructs, LVO helped 
with cytological analysis and prepared a draft of the manuscript, and LSC was the principal investigator of the project and participated in the design, coordination, and writing of the manuscript. All authors read and approved the final manuscript.

\section{Acknowledgements}

We sincerely thank Rick G. Fehon for providing us various Merlin mutant strains and the anti-Merlin antibody. We are also grateful to Alain Debec for the strain with GFP insertion in the CG835 I gene, and lgor Zhimulev for the Delta2-3 strain. Special appreciation goes to Cynthia McAllister for EM assistance, and Amanda Simcox and Sarah S. Burns for critical reading of the manuscript. This study was supported by grants from the US Department of Defense Neurofibromatosis Research Program (W8IXWH-04-I-0509) and Russian Fund of Fundamental Investigation (05-04-483I6).

\section{References}

I. Lindsley DL, Tokuyasu KT: Spermatogenesis. In Genetics and Biology of Drosophila 2nd edition. Edited by: Ashburner M, Wright TR. Academic Press, NY, NY; 1980:225-294.

2. Fuller M: Spermatogenesis. In The Development of Drosophila melanogaster Edited by: Bate M, Arias AM. Cold Spring Harbor Laboratory Press, NY; 1993:7|-147.

3. Cross DP, Shellenbargerr DL: The dynamics of Drosophila melanogaster spermatogenesis in in vitro cultures. J Embryol Exp Morph 1979, 53:345-35I.

4. Fabrizio J, Hime G, Lemmon S, Bazinet C: Genetic dissection of sperm individualization in Drosophila melanogaster. Development 1998, I 25: 1833-1843.

5. Fuller MT: Genetic control of cell proliferation and differentiation in Drosophila spermatogenesis. Semin Cell Dev Biol 1998, 9:433-444.

6. Rouleau GA, Merel P, Luchtman M, Sanson M, et al.: Alteration in a new gene encoding a putative membrane-organizing protein causes neurofibromatosis type 2. Nature 1993, 363:515-52I.

7. Trofatter JA, MacCollin MM, Rutter JL, Murrell JR, et al.: A novel Moesin-, Exrin-, Radixin-like gene is a candidate for the neurofibromatosis 2 tumor-suppressor. Cell 1993, 72:79|-800.

8. Lajeunesse DR, McCartney BM, Fehon RG: Structural analysis of Drosophila merlin reveals functional domains important for growth control and subcellular localization. J Cell Biol 1998, 141:1589-1599.

9. Algrain M, Arpin M, Louvard D: Wizardry at the cell cortex. Current Biol 1993, 3:45I-454.

10. Bretscher A, Edwards K, Fehon RG: ERM proteins and merlin: integrators at the cell cortex. Nat Rev Mol Cell Biol 2002, 3:586-599.

II. Okada T, You L, Giancotti FG: Shedding light on Merlin's wizardry. Trends Cell Biol 2007, 17:222-229.

12. Xu HM, Gutmann DH: Merlin differentially associates with the microtubule and actin cytoskeleton. J Neurosci Res 1998, 5 I:403-4I5.

13. Muranen T, Gronholm M, Lampin A, Lallemand D, Zhao F, Giovannini $M$, Carpen $O$ : The tumor suppressor merlin interacts with microtubules and modulates Schwann cell microtubule cytoskeleton. Hum Mol Genet 2007, 16: 1742-I75।.

14. Lallemand D, Curto M, Saotome I, Giovannini M, McClatchey AI: NF2 deficiency promotes tumorigenesis and metastasis by destabilizing adherens junctions. Genes Dev 2003, I7:1090-I I 00.

15. McClatchey Al, Giovannini M: Membrane organization and tumorigenesis - the NF2 tumor suppressor, Merlin. Genes Dev 2005, 1 9:2265-2267.

16. Golovnina K, Blinov A, Akhmametyeva EM, Omelyanchuk LV, Chang LS: Evolution and origin of merlin, the product of the Neurofibromatosis type 2 (NF2) tumor-suppressor gene. BMC Evol Biol 2005, 5:69-86.

17. McCartney BM, Fehon RG: Distinct cellular and subcellular patterns of expression imply distinct functions for the Drosophila homologues of moesin and the neurofibromatosis 2 tumor suppressor, merlin. J Cell Biol 1996, 133:843-852.
18. Chishti AH, Kim AC, Marfatia SM, Lutchman M, et al:: The FERM domain: a unique module involved in the linkage of cytoplasmic proteins to the membrane. Trends Biochem Sci 1998, 23:28I-282.

19. Fehon RG, Oren T, Lajeunesse DR, Melby TE, McCartney BM: Isolation of mutations in the Drosophila homologues of the human Neurofibromatosis 2 and yeast CDC42 genes using a simple and efficient reverse-genetic method. Genetics 1997, 146:245-252.

20. Hamaratoglu F, Willecke M, Kango-Singh M, Nolo R, Hyun E, Tao C, Jafar-Nejad H, Halder G: The tumour-suppressor genes NF2/ Merlin and Expanded act through Hippo signaling to regulate cell proliferation and apoptosis. Nat Cell Biol 2006, 8:27-36.

21. Cho E, Feng Y, Rauskolb C, Maitra S, Fehon R, Irvine KD: Delineation of a Fat tumor suppressor pathway. Nat Genet 2006, 38: I 142-II 50.

22. Silva E, Tsatskis Y, Gardano L, Tapon N, McNeill H: The tumor-suppressor gene fat controls tissue growth upstream of expanded in the hippo signaling pathway. Current Biol 2006, 16:208I-2089.

23. Pellock BJ, Buff E, White K, Hariharan IK: The Drosophila tumor suppressors Expanded and Merlin differentially regulate cell cycle exit, apoptosis, and Wingless signaling. Dev Biol 2007, 304: 102-115.

24. Maitra S, Kulikauskas RM, Gavilan H, Fehon RG: The tumor suppressors Merlin and Expanded function cooperatively to modulate receptor endocytosis and signaling. Current Biol 2006, 16:702-709.

25. Curto M, Cole BK, Lallemand D, Liu CH, McClatchey Al: Contactdependent inhibition of EGFR signaling by Nf2/Merlin. J Cell Biol 2007, 177:893-903.

26. MacDougall N, Lad Y, Wilkie G, Francis-Lang H, Sullivan W, Davis I: Merlin, the Drosophila homologue of nerurofibromatosis-2, is specifically required in posterior follicle cells for axis formation in the oocyte. Development 200I, I 28:665-673.

27. Ashburner M: Drosophila: a laboratory manual. Cold Spring Harbor Laboratory Press, NY; 1989.

28. Jin H, Sperka T, Herrlich P, Morrison H: Tumorigenic transformation by CPI- 17 through inhibition of a merlin phosphatase. Nature 2006, 442:576-579.

29. Giansanti MG, Farkas RM, Bonaccorsi S, Lindsley DL, Wakimoto BT, Fuller MT, Gatti M: Genetic dissection of meiotic cytokinesis in Drosophila males. Mol Biol Cell 2004, I 5:2509-2522.

30. Gateff E: Tumor suppressor and overgrowth suppressor genes of Drosophila melanogaster: Developmental aspects. Int jDev Biol 1994, 38:565-590.

31. Kang-Decker N, Mantchev GT, Juneja SC, McNiven MA, van Deursen JMA: Lack of acrosome formation in Hrb-deficient mice. Science 200I, 294: I53I-I533.

32. Yao R, Ito $C$, Natsume $Y$, Sugitani $Y$, Yamanaka $H$, et al:: Lack of acrosome formation in mice lacking Golgi protein GOPC. Proc Natl Acad Sci USA 2002, 99: I I 2 I I-II 216.

33. Kierszenbaum AL, Tres LL, Rivkin E, Kang-Decker N, van Deursen JM: The acroplaxome is the docking site of Golgi-derived myosin $\mathrm{Va} / \mathrm{Rab} 27 \mathrm{a} / \mathrm{b}$-containing proacrosomal vesicles in wild-type and Hrb mutant mouse spermatids. Biol Reprod 2004, 70:1400-1410.

34. Kierszenbaum AL, Rivkin E, Tres LL: The actin-based motor myosin $\mathrm{Va}$ is a component of the acroplaxome, an acrosome-nuclear envelope junctional plate, and of manchetteassociated vesicles. Cytogenet Genome Res 2003, 1 03:337-344.

35. Kierszenbaum AL, Rivkin E, Tres LL: Acroplaxome, an F-actinkeratin-containing plate, anchors to the acrosome to the nucleus during shaping of the spermatid head. Mol Biol Cell 2003, I 4:4628-4640.

36. Noguchi T, Miller KG: A role for actin dynamics in individualization during spermatogenesis in Drosophila melanogaster. Development 2003, 130:1805-1816.

37. Hales KG, Fuller MT: Developmentally regulated mitochondrial fusion mediated by a conserved, novel, predicted GTPase. Cell 1997, 90:121-129.

38. Bergeret E, Pignot-Paintrand I, Guichard A, Raymond K, Fauvarque MO, Cazemajor M, Griffin-Shea R: RotundRacGAP functions with Ras during spermatogenesis and retinal differentiation in Drosophila melanogaster. Mol Cell Biol 200I, 21:6280-629I. 
39. Yamada D, Yoshida M, Williams YN, Fukami T, et al.: Disruption of spermatogenic cell adhesion and male infertility in mice lacking TSLCI/IGSF4, an immunoglobulin superfamily cell adhesion molecule. Mol Cell Biol 2006, 26:3610-3624.

40. Lajeunesse DR, McCartney BM, Fehon RG: A systematic screen for dominant second site modifiers of Merlin/NF2 phenotypes reveals an interaction with blistered/DSRF and scribbler. Genetics 200I, I 58:667-679.

Publish with Bio Med Central and every scientist can read your work free of charge

"BioMed Central will be the most significant development for disseminating the results of biomedical research in our lifetime. " Sir Paul Nurse, Cancer Research UK

Your research papers will be:

- available free of charge to the entire biomedical community

- peer reviewed and published immediately upon acceptance

- cited in PubMed and archived on PubMed Central

- yours - you keep the copyright

Submit your manuscript here:

http://www.biomedcentral.com/info/publishing_adv.asp
BioMedcentral 